\title{
THE NEWPOSSIBILITIES OF SUPERLANS CODE FOR EVALUATION OF AXISYMMETRIC CAVITIES
}

\author{
D.G.Myakishev, V.P.Yakovlev, Budker INP, 630090 Novosibirsk, Russia
}

The new versions of the SuperLANS code for evaluation of monopole modes in axisymmetric cavity with lossy filling and of multipole modes in axisymmetric cavity with inhomogeneous ferrite and dielectric filling are presented.

\section{INTRODUCTION}

The code SuperLANS [1] is developed in two directions:

- a code CLANS is made to calculate monopole modes in axisymmetric cavities with partial lossy dielectric and ferrite filling;

- a code SLANS2 is made to calculate multipole modes in axisymmetric cavities with partial dielectric and ferrite filling.

\section{CLANS}

Being the new version of SuperLANS, the code CLANS is developed to calculate monopole modes in axisymmetric cavities with lossy dielectric and ferrite filling, providing:

- evaluation of a cavity with dielectric and ferrite with loss tangent $\operatorname{tg} \delta \leq 1$;

- solution of self-consistent problem for frequency dependent dielectric permittivity and magnetic permeability.

\section{A. Mathematical formulation}

To describe lossy filling with loss tangent $\operatorname{tg} \delta \leq 1$, the complex permittivity and permeability are used. The electric and magnetic fields are also complex. The equation for the magnetic field, for example, for TM wave, is the same one as for SuperLANS [1]:

$$
\frac{1}{\varepsilon}(\Delta \vec{H})_{\varphi}+\omega^{2} \varepsilon_{0} \mu_{0} \mu \cdot \vec{H}_{\varphi}=0
$$

The equation of such form provides the boundary condition on the dielectric and ferrite surfaces [2]. We use the coordinate system $\left(\mathrm{z}, \mathrm{r}^{2} / 4\right)$ as in SuperLANS to improve solution near axis.

\section{B. Solution method}

The equation (1) is solved by the finite element method. We use four corner eight nodes isoparametric elements. Algebraic system of equations for node field values is produced by Galerkin method. To find several modes simultaneously in arbitrary spectrum domain, we use subspace iteration method with frequency shift.

If the permittivity and permeability of lossy filling depend on frequency, the self-consistent problem is solved. The iteration is produced in the next way. The solution for fixed permittivity and permeability is used as an initial approach. Then for this frequency the new values of permeability and permittivity are chosen. Using this values of permittivity and permeability, we find the new frequency by the method of inverse iteration with frequency shift, which provides the effective separation of searched mode. We use the frequency of the previous iteration as a shift. As a rule, several iterations are enough to obtain the selfconsistent solution. Iterations are produced automatically, if a file with permittivity and permeability frequency dependencies is prepared.

There are two versions of code for PC and VAX at present.

\section{Results.}

The code was tested on pillbox cavity with homogeneous losses filling. For cavity with sizes $r=1 \mathrm{~cm}$ and $l=1 \mathrm{~cm}$ with filling material permittivity and permeability $\varepsilon=1, \mu=1$ and loss tangent $\operatorname{tg} \delta=1$, with mesh $5 * 5$ the fundamental mode frequency calculation accuracy is $0.01 \%$.

The VAX version of the code is used for calculation of superconducting cavity with ferrite HOM damper in Cornell University [3]. The force lines of real and image parts of electric field for fundamental modes are shown on Fig. 1 and Fig.2. The force lines of real and image parts of electric field for "ghost" mode are shown on Fig. 3 and Fig.4. This mode is obtained as a solution of self-consistent problem and connects to ferrite loading.

\section{SLANS2}

The code SLANS2 is developed to calculate the multipole modes in axisymmetric cavities with partial dielectric and ferrite filling.

\section{A. Mathematical formulation}

Unlike the scalar problem for monopole modes the problem of multipole mode calculation is a vector problem. In our case we can write usual wave equation, for example, for magnetic field:

$$
\operatorname{rot} \frac{1}{\varepsilon} \operatorname{rot} \vec{H}-\omega^{2} \varepsilon_{0} \mu_{0} \mu \cdot \vec{H}=0
$$

To eliminate spurious modes we additionally use the equation:

$$
\operatorname{grad} \cdot \operatorname{div}(\mu \vec{H})=0
$$

Combining (2) and (3), we obtain the following equation for partially homogeneous filling:

$$
\frac{1}{\varepsilon}(\operatorname{rot} \cdot \operatorname{rot} \vec{H}-\mathrm{grad} \cdot \operatorname{div} \vec{H})-\omega^{2} \varepsilon_{0} \mu_{0} \mu \cdot \vec{H}=0
$$


Rewriting this equation for field components and excluding $\varphi$-component using (3), we obtain two equations for $\mathrm{Hz}$ and $\mathrm{Hr}$ without the potential solutions [4], [5].

Boundary conditions for tangential and normal components of magnetic field on a metallic surface have the following form [6]:

$$
\frac{\partial H_{\tau}}{\partial n}+K \cdot H_{\tau}=0, \quad H_{n}=0
$$

where $\mathrm{K}$ - is surface curvature in the axial cross section.

Solving eigen value problem for two transverse components of magnetic field we can reconstruct $\varphi$ component of magnetic field and all components of electric field from Maxwell's equations.

SLANS2 also permits to solve eigen value problem for transverse components of electric field. In this case equations have the same form, except boundary conditions on a metallic surface, which have the following form [6]:

$$
\frac{\partial E_{n}}{\partial n}+\left(K+\frac{n_{r}}{r}\right) E_{n}=0, \quad E_{\tau}=0
$$

where $n_{\mathrm{r}}$ is a radial component of the unit vector normal to the boundary.

Unlike SuperLANS and CLANS, we use the usual coordinate system $(\mathrm{z}, \mathrm{r})$ for SLANS2.

\section{B.Solution method}

To produce finite element mesh, we used the same mesh generator as for SuperLANS. Galerkin method is used to obtain the algebraic system. Unlike SuperLANS the matrix of system is not symmetric, so we must store the whole band of matrix.

To satisfy the boundary condition on a metallic surface (5) we use the method described in [6]. We rewrite discrete equations on metallic surfaces for normal and tangential field components: $H n, H \tau$ or $E n, E \tau$. For this, we use a local matrix of rotation for the node field values on metal.

On ferrite or dielectric surfaces the problem of satisfying the boundary condition is more complicated. Only the tangent field components $H \tau$ and $E \tau$ are continuous and the normal components $H n$ and $E n$ have break. So we rewrite the discrete equations for $B n, H \tau$ or $D n, E \tau$ on ferrite or dielectric surfaces to satisfy the boundary conditions. This method permits to use a regular finite element mesh as in SuperLANS.

To calculate several modes simultaneously in arbitrary spectrum domain, we use the subspace iteration method.

\section{C.Results}

The spherical cavity with concentric spherical dielectric or ferrite insertion was used as a test. The convenience of this test is that it is possible to compare calculation results of SuperLANS and SLANS2, because monopole modes of SuperLANS are dipole modes of SLANS2 rotated by $90^{\circ}$. For the same meshes the differences between SuperLANS and SLANS2 results are less than $0.015 \%$.
The code SLANS2 is used to calculate cavities for RF generator Magnicon [7]. The operating modes in this device are multipole. The field maps for two different modes for Magnicon penultimate cavity are shown on Fig.5 and Fig.6.

The code was also used to calculate a variant of the waveguide window for VLEPP klystron [8]. The window consist of a ceramic disk, which attached to a rectangular waveguide by the conical parts. The dipole modes are excited in such axisymmetric cavity. The field maps for two different modes are shown on Fig.7 and Fig.8: the first one is the "ghost" mode, which exist in the ceramic disk only, the second mode is one of the propagation modes.

\section{CONCLUSIONS}

The codes CLANS and SLANS2, which are the advancement of the SuperLANS code for axisymmetric cavities, have proven to be very powerful tools for the analysis of many RF design problems.

Futher development will be creation of the code for calculation of multipole oscillation in cavities with lossy filling.

\section{REFERENCES}

[1] D.G.Myakishev, V.P.Yakovlev, "An Interactive Code SuperLANS for Evaluation of RF-cavities and Acceleration Structures", in IEEE Particle Accelerator Conf. Rec.,1991, vol.-5, pp.3002-3004.

[2] M.M.Karliner, Yu.A.Sokulin, V.P.Yakovlev, "Calculation of azimuthal oscillation in axisymmetric cavities with nonhomogeneous ferrite and dielectric filling." preprint Budker INP 82-149 (in Russian).

[3] S.Belomestnykh, W.Hartung, G.Flynn, H.Padamsee, M.Pisharody, "Wakefields and HOMs Studies of a Superconducting Cavity Module with the CESR Beams", report at present conference.

[4] M.M.Karliner, B.M.Fomel, V.P.Yakovlev, "LANS2 code for calculation of azimuthally nonhomogeneous oscillation in axisymmetric." preprint Budker INP 83-114 (in Russian).

[5] M.S.Kaschiev, V.A.Kaschieva, I.V.Puzynin, V.V.Gusev, A.I.Fedoseev, I.V.Gonin, V.V.Paramonov, "Calculation of the total oscillation spectrum in axisymmetrical RF cavities and periodic structures", in Proc.IX All-Union Conf. Particle Accelerators, Dubna, USSR,1984, vol. I, pp.137-141 (in Russian).

[6] Yu.Portugalov, "Method of eigen electromagnetic field calculation in cavities of arbitrary shape", preprint IHEP 83-2, Serpukhov (in Russian).

[7] O.A.Nezhevenko, "The magnicon: A new RF power source for accelerators" in IEEE Particle Accelerator Conf. Rec., 1991, vol.-5, pp.2933-2942.

[8] V.Shemelin, "A choice of high power RF window sizes", Int.Workshop on Pulsed RF Power Sources for Linear Colliders, Dubna-Protvino, 1993, BINP July 1993, pp. 369-372. 


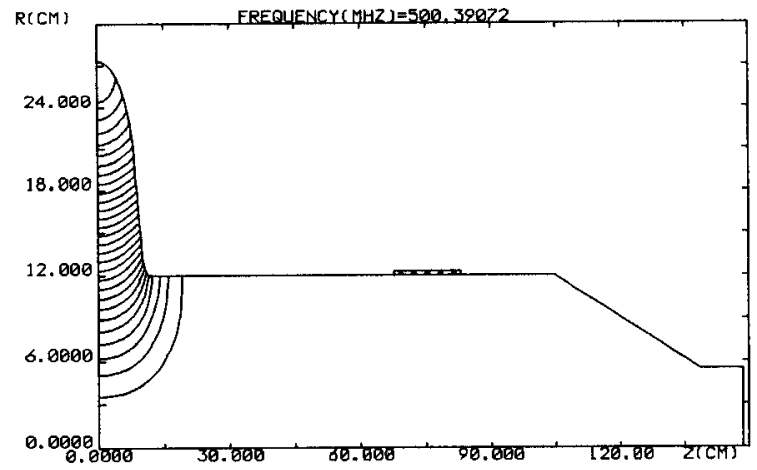

Figure 1: Real part of electric field of the operating mode of superconducting cavity.

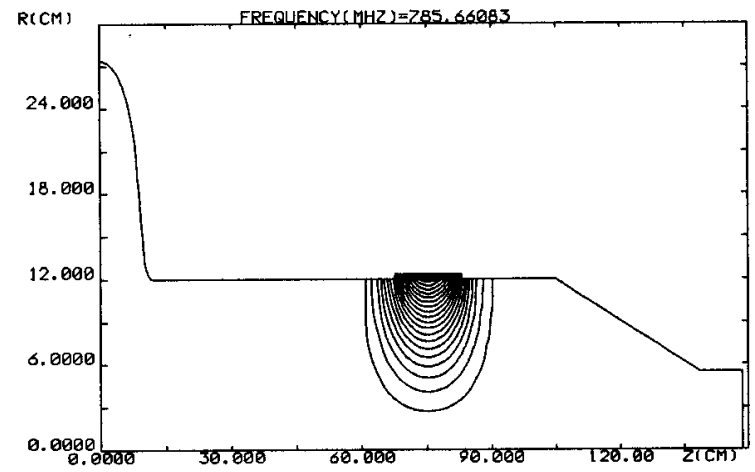

Figure 3: Real part of electric field of the "ghost" mode of superconducting cavity.

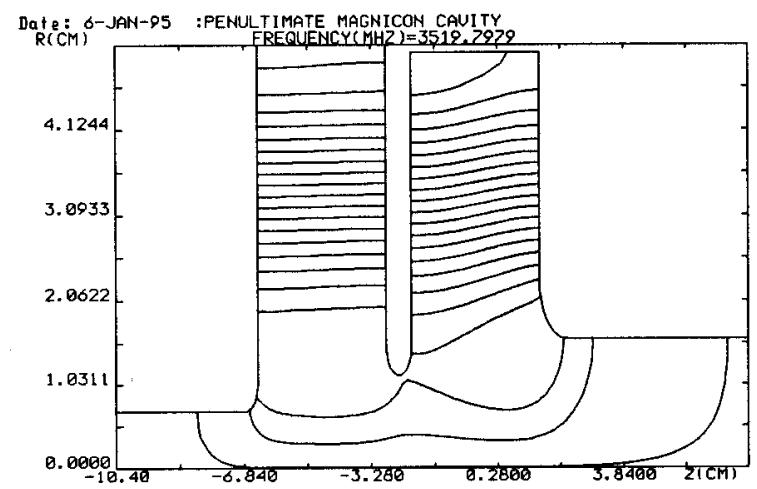

Figure 5: Field map of cophasal mode of the Magnicon penultimate cavity.

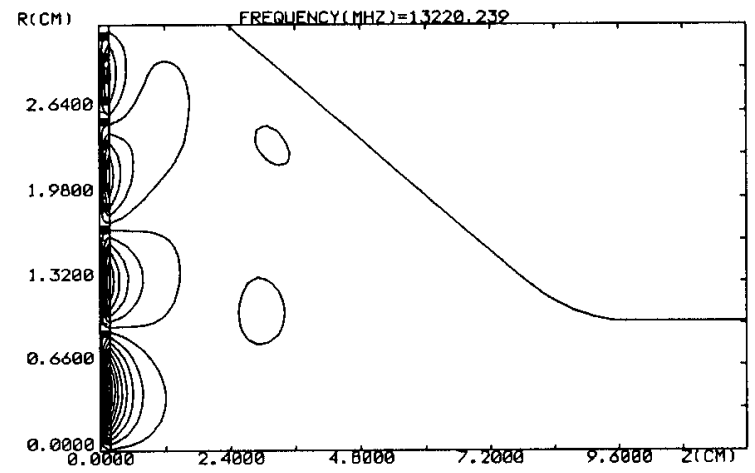

Figure 7: Field map of "ghost"mode in RF-window.

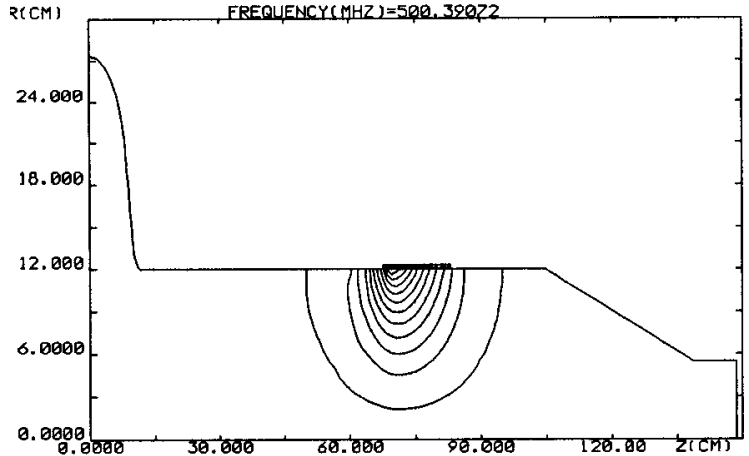

Figure 2: Imaginary part of electric field of the operating mode of superconductive cavity.

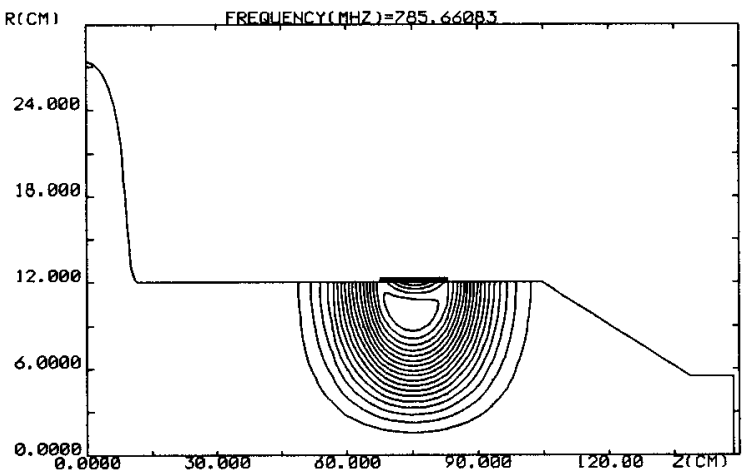

Figure 4: Imaginary part of electric field of the "ghost" mode of superconductive cavity.

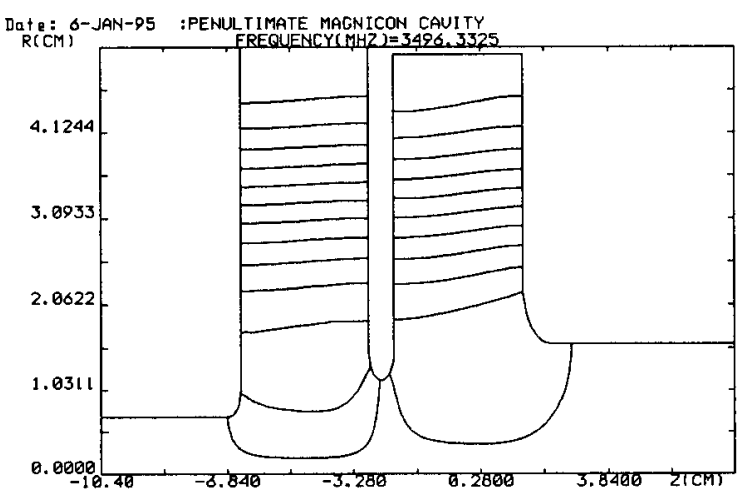

Figure 6: Field map of counterphasal mode of the Magnicon penultimate cavity.

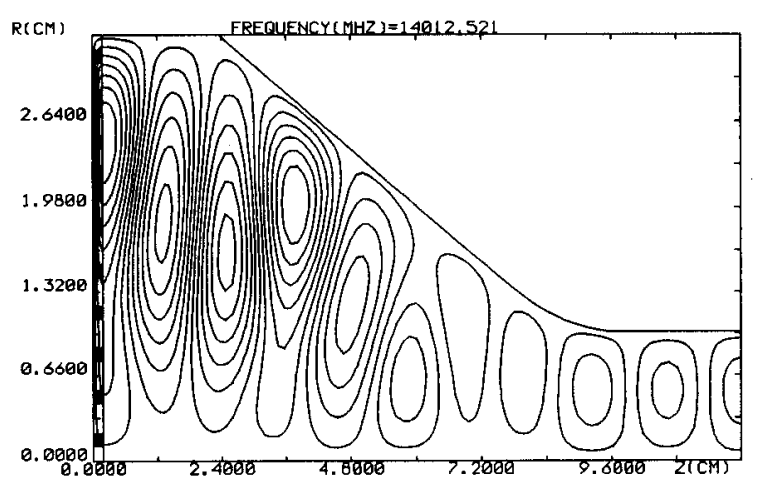

Figure 8: Field map of propagation mode in RF- window. 